\title{
LOS CAMBIOS INTERMODÉLICOS EN LOS PROGRAMAS DE INVESTIGACIÓN IDEALISTA DE LA LINGÜÍSTICA
}

\author{
JUAN LUIS JiMÉNEZ RUIZ \\ Universidad de Alicante \\ Jimenez@ua.es
}

\begin{abstract}
Resumen
La reflexión epistemológica sobre la Lingüística posee un componente sincrónico, la Filosofia de la ciencia lingüística, y un componente diacrónico, la Historiografia de la Lingüistica, en la que se realizan dos tipos de análisis sobre el cambio en los programas de investigación: 1, el cinemático, describiendo las entidades involucradas en los cambios y los tipos y formas de los mismos; y 2, el dinámico, identificando las causas y factores desencadenantes del cambio. Mientras el análisis opositivo se centra en el primero, intentando precisar la identidad del cambio, el acercamiento lineal, centrado en el segundo, pretende estudiar la problemática de la continuidad a través de la ruptura. Es lo que hacemos en este trabajo, acercándonos linealmente al paradigma idealista de la Lingüística desde Platón hasta la Filosofía de la ciencia de tradición Feyerabendiana.

PALABRAS CLAVE: Filosofia de la Lingüística, Historia de la Lingüística, Filosofia del lenguaje, Conocimiento.
\end{abstract}

\begin{abstract}
The discipline of epistemology of linguistics is divided into a synchronic component, philosophy of linguistic, \& a diachronic component, historiography of linguistics, the latter addresses changes in patterns of linguistics research through two types of analysis: (1) cinematic, describing entities involved in changes \& types \& forms of changes, \& (2) dynamic, identifying causes \& triggers of change. Whereas the oppositive approach based on (1) attempts to account for identity through change, the linear approach based on (2) investigates continuity across breaches or disjuncture. In this framework, the idealist paradigm in the philosophy of language can be traced from Platon through the philosophy of science in the Feyerabend tradition.
\end{abstract}

KEY WORDS: Philosophy of Linguistic, Linguistic History, Philosophy of Language, Knowledge.

\section{Los orígenes de los programas de investigación idealista}

Tal y como hicimos anteriormente con el Paradigma realista de la Lingüística (Jiménez Ruiz, 2005: 275-299), vamos ahora a esbozar una breve panorámica de cómo se forjó la reflexión epistemológica idealista de la Lingüística. Recordemos que seguimos con un acercamiento lineal que, a partir del estudio dinámico, tiene por objetivo la resolución de la problemática de la continuidad a través de la ruptura. Para ello, vamos a poner en relación cada uno de los distintos momentos de los ciclos que establecimos en otra ocasión (Jiménez Ruiz, 2004: 895-907), con el fin de comprender en su justa medida la reelaboración lingüística evolutiva (cambio intermodélico).

Los origenes de la reflexión idealista se remontan ahora a Platón, quien en el Crátilo intenta liberar el pensamiento de la estrecha relación entre la palabra y la cosa proyectándolo 
sobre el eidos y buscando el logos en la antagónica estructura de nomos y phisis puesto que las palabras no ofrecían la naturaleza de su objeto. Se inicia así la concepción simbólica del lenguaje, que es representación de nosotros mismos, y después de las cosas. En este sentido, no interesa tanto el hecho de que las palabras muestren las cosas, sino el procedimiento interno que permite esta mostración. Se trata de un procedimiento de justificación del fenómeno lingüístico desde el ámbito teórico de la lengua como objeto empírico, como vehículo de expresión de la trascendencia del lenguaje sujeto, incognoscible directamente por su inmaterialidad (Jiménez Ruiz, 1994: 128 y ss.).

Esta reflexión dio lugar a la teoría phisey, según la cual las palabras designan las cosas según su naturaleza. Tal postulado defiende la concepción de un lenguaje no sometido a reglas, que es producto de la Naturaleza y, como ésta, no sujeto a ninguna imposición categorial. Por todo ello, el nombre es, como en la pintura, una imitación del objeto, una imagen empírica de una realidad trascendente.

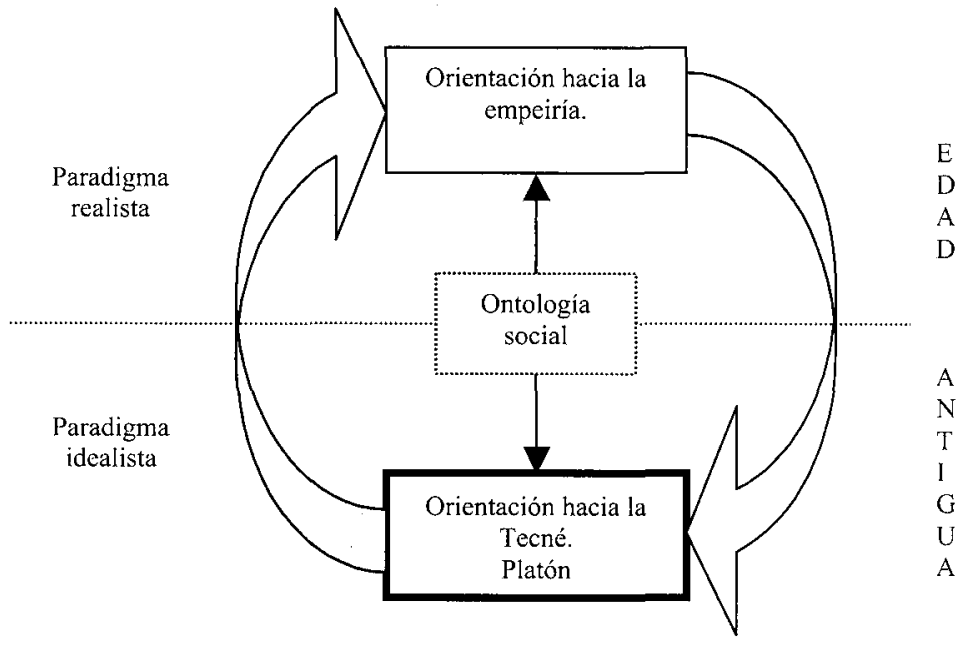

\section{El idealismo epistemológico durante la Edad Media}

Durante la Edad Media las investigaciones lingüísticas fueron realizadas por gramáticos que sustentaban sus reflexiones en una visión filosófica del mundo y, consecuentemente, del lenguaje (Jiménez Ruiz, 2001: 124). En el fondo se trataba más que de gramáticos, de filósofos que aplicaban sus conocimientos filosóficos a los estudios lingüísticos, identificando las categorías lógicas con las lingüísticas; de ahí que el substantivo portase substancia, el adjetivo cualidad o el verbo movimiento, por poner unos casos, cuando en realidad el valor funcional de las formas lingüísticas se precisa gracias a las relaciones sintagmáticas y paradigmáticas.

En este sentido, se sigue reflexionando sobre el lenguaje, ahora desde un punto de vista lógico, sirviendo la lengua para las especulaciones filosóficas. 


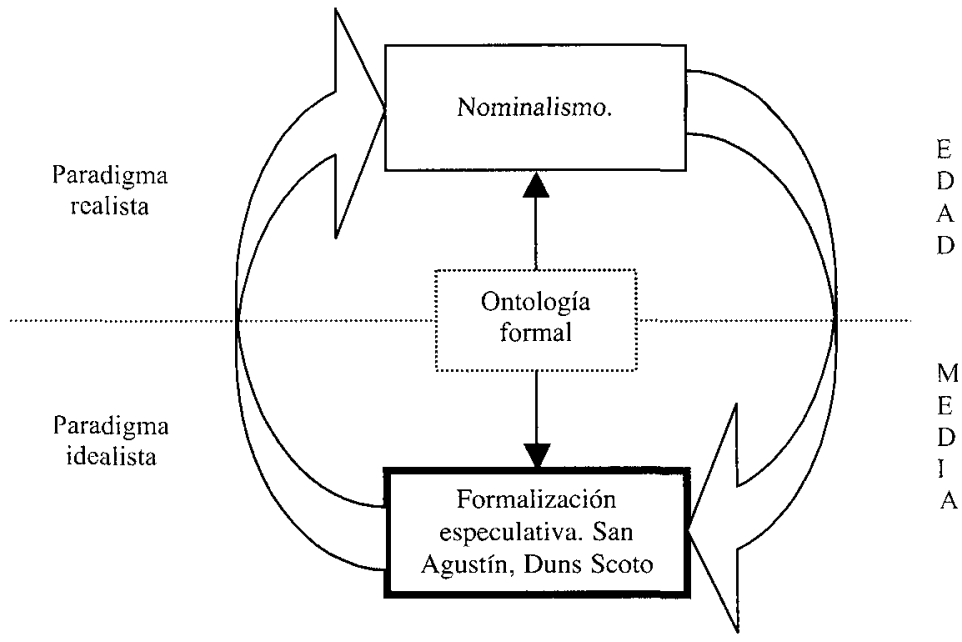

Por ello, estos planteamientos tendrán su desarrollo en la especulación medieval (San Agustín, Duns Scoto) que, aunque sostiene que el hombre está compuesto por forma y materia, la forma específica del hombre es el alma, que posee su propia naturaleza. Por tanto, la explicación cognoscitiva se sitúa en el alma, que busca conocer la realidad (Monserrat, 1984: 17).

En el terreno lingüístico, la realidad estaba representada por una supremacía del latín, que era la lengua de cultura, el estudio y la Iglesia. Por ello, se elaboran manuales que siguen a los autores romanos Donato y Prisciano.

Entre los principales estudios éncargados del análisis del latín destacan la Gramática de Alcuino de York (siglo vII y vIII); las Etimologías de San Isidoro (siglo vII); el Primer Tratado Gramatical de un autor irlandés desconocido del siglo xil; y los Doctrinale de Alejandro de Villedieu (escrito en verso en el año 1200), que perduró hasta 1588, por poner unos casos.

Estas investigaciones se van a caracterizar por dos rasgos principalmente: la interrogación filosófica sobre los hechos lingüísticos y el dominio del criterio de la autoridad y la fe en todo el quehacer intelectual.

\section{Los programas de investigación idealista durante la Modernidad}

Durante la Modernidad (Herder) y precisamente como una reacción contra el Sensismo - Teoría del conocimiento que pretende reducir todo el proceso cognitivo a la información proporcionada por los sentidos-, el Fenomenalismo - que sostiene que la información la proporciona la cosa - y el Empirismo - que sostiene que el proceso cognoscitivo deriva de la experiencia-, se defiende que el conocimiento es un hecho humano fundamental, analizado y explicado de distinta manera según la óptica adoptada, ya que el conocimiento es, en última instancia, reconocimiento «en lo otro, en lo otro de los hombres, en lo otro del acontecer» (Koselleck y Gadamer, 1997: 105). 


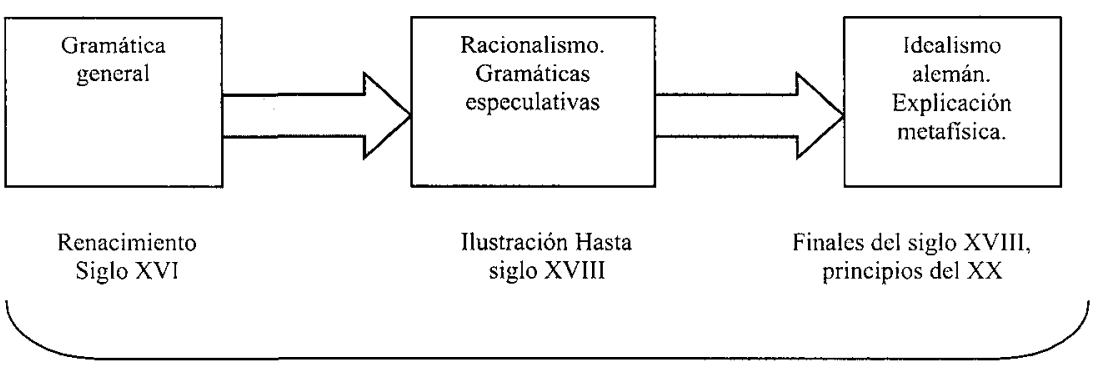

EDAD MODERNA

Así pues, el lenguaje no será ya un instrumento, sino que el acto de pensar mismo será ya un acto de lenguaje, y puesto que el hombre es un ser activo y con libertad de pensar, será una criatura de lenguaje ${ }^{1}$. Desde este punto de vista, el Lenguaje se convierte en el creador del hombre mismo, en la determinación de la energía del Espíritu. De esta manera, de órgano, el lenguaje se ha convertido en organismo: es el creador del hombre mismo, es un modo y una determinación de la energía del espíritu.

Ello se traducirá en las formalizaciones lingüísticas prescriptivas del siglo xvı (Gramática general), especulativas del xvi y principios del xviII (Descartes) y metafísicas de finales del XVIII y principios del XIX (Hegel).

\subsection{Las Gramáticas generales del Renacimiento}

La Edad Moderna va a propiciar también en el período renacentista la búsqueda de una Gramática general.

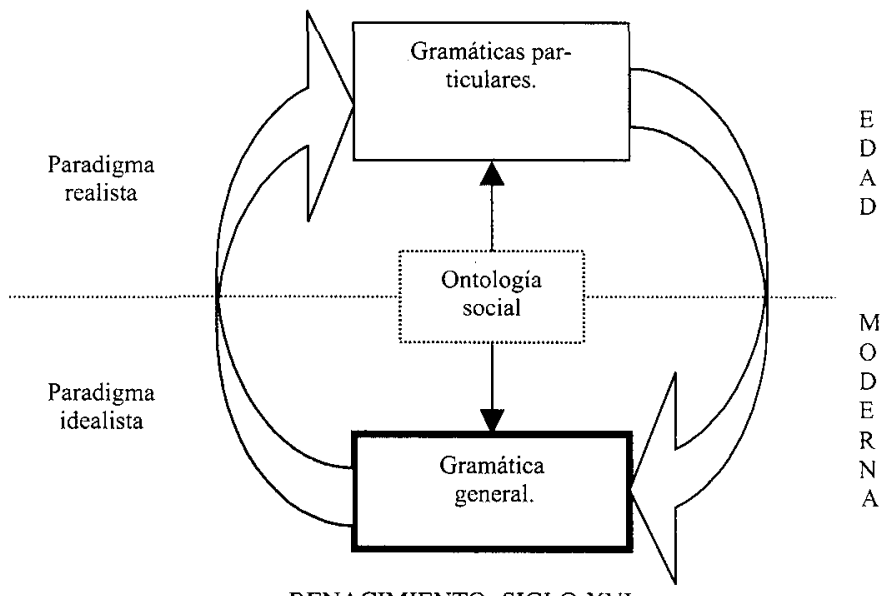

RENACIMIENTO: SIGLO XVI

1 De hecho, se potencia así la dimensión hermenéutica del propio pensamiento, que será puesta posteriormente de relieve por Gadamer - basada en la concepción platónico hegeliana-. De aquí surge precisamente la necesidad de reivindicar la dimensión ontológica de la memoria. Sobre esta tesis puede verse el trabajo de Esteban Ortega (1996: 403-428). 
Como manifestamos con anterioridad (Jiménez Ruiz, 2001: 128), fue principalmente el carácter universal de las categorías lógicas y la identificación de las mismas con las categorías lingüísticas, los que posibilitaron la creencia también de un universalismo en el terreno lingüístico y, consecuentemente, la búsqueda de una Gramática general (universal), válida para todas las lenguas. Fruto de ello fue, por ejemplo, la creencia de que el español tenía declinaciones.

Se trata de una Gramática general, universal o filosófica que estudiará el lenguaje orientado a su dimensión cognitiva y representacional, a partir de los siguientes presupuestos:

- el concepto de evolución diacrónica de las lenguas y el parentesco entre las mismas.

- La importancia de la fonética porque se quiere conocer bien el latín.

- Al estudiarse las lenguas cultas y populares surge la cuestión de su parentesco. Se consideran causas de la evolución lingüística los factores de contacto entre lenguas y la evolución interna de las mimas.

\subsection{La investigación racionalista}

Con todo, será con el Racionalismo (Descartes) cuando se vincule la Lingüística a las gramáticas especulativas como paso previo a las formulaciones transformatorias americanas, buscando la seguridad frente a las inseguridades del empirismo ${ }^{2}$.

Tal y como hicimos anteriormente con el Empirismo (Jiménez Ruiz, 2005: 283-285), vamos a repasar ahora los puntos fundamentales de la Epistemología racionalista (Monserrat, 1984: 19):

- La convicción de que la experiencia no es la única fuente de conocimiento y de que las sensaciones no bastan para dar origen a los conocimientos si éstos no son trabajados por la razón. Por ello, la conciencia será así activa, creadora, gracias a la razón.

- La certeza de que la razón, radicada en el espíritu del hombre, tiene una potencia que la constituye en fuente del conocimiento real, puesto que todo lo racional es real y todo lo real es racional.

- Y, finalmente, la seguridad de que junto a las verdades de hecho - aquellos contenidos reales cuya verdad conocemos por la experiencia- existen las verdades de razón - contenidos reales que, en este caso, conocemos por medio de la razón-. 


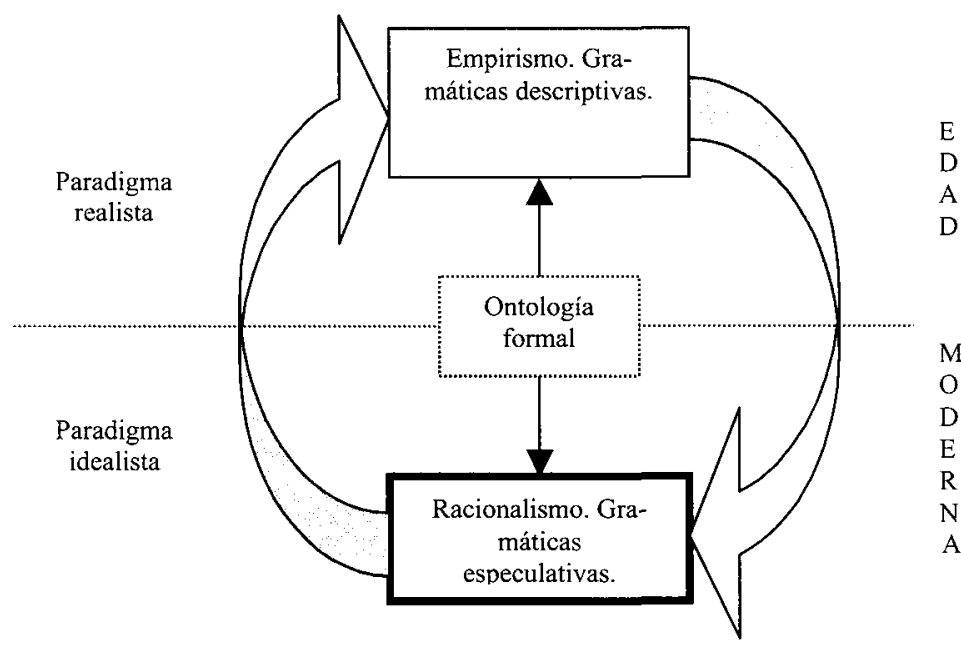

ILUSTRACIÓN: HASTA SIGLO XVIII

Para que se produzca este conocimiento, Descartes (1990: 16) estima que hay que aplicar cuatro preceptos, que deben ser siempre tenidos en cuenta:

«El primero consistía en no admitir jamás cosa alguna como verdadera sin haber conocido con evidencia que así era; es decir, evitar con sumo cuidado la precipitación y la prevención y no admitir en mis juicios nada más que lo que se presentase tan clara y distintamente a mi espíritu que no tuviese motivo alguno ara ponerlo en duda.

El segundo, en dividir cada una de las dificultades a examinar en tantas partes como fuera posible y necesario para su mejor solución.

El tercero, en conducir con orden mis pensamientos, empezando por los objetos más simples y más fáciles de conocer, para ascender poco a poco, gradualmente, hasta el conocimiento de los más complejos, y suponiendo incluso un orden entre aquellos que no se preceden naturalmente unos a otros.

$Y$ el último, en hacer en todo enumeraciones tan completas y revisiones tan amplias, que llegase a estar seguro de no haber omitido nada.

Por todo ello, para la Epistemología racionalista el hombre podrá formular conocimientos universales puesto que la razón tiene la facultad de entender lo que se da en la realidad necesariamente, frente al Empirismo que nunca podrá justificar esa necesidad.

\subsection{La aportación del Idealismo alemán}

Hacia finales del siglo xVIII y principios del XIX los filósofos del Idealismo alemán - sobre todo Hegel- contribuyeron a la Epistemología idealista con una reflexión sobre la naturaleza y la realidad empírica basada en sus propios sistemas metafísicos.

Hegel (1964: 51 y ss.) sostiene que para conocer la verdad de la realidad hay que hacerlo desde la reflexión introspectiva. Este proceso le manifiesta que en la conciencia — cuando se 
produce el conocimiento- existen dos elementos; a saber, la esencia del objeto y el saber acerca del objeto, cuyo conjunto recibe el nombre de figura-de-conciencia.

Como sostienen Díez y Moulines (1999: 29), los epistemólogos idealistas de este período se mostraron muy escépticos y opuestos al espíritu de la ciencia empiricomatemática, tal y como se desarrolló a partir del siglo XVII. Por ello, formularon un programa alternativo a la ciencia entendida en el sentido moderno.

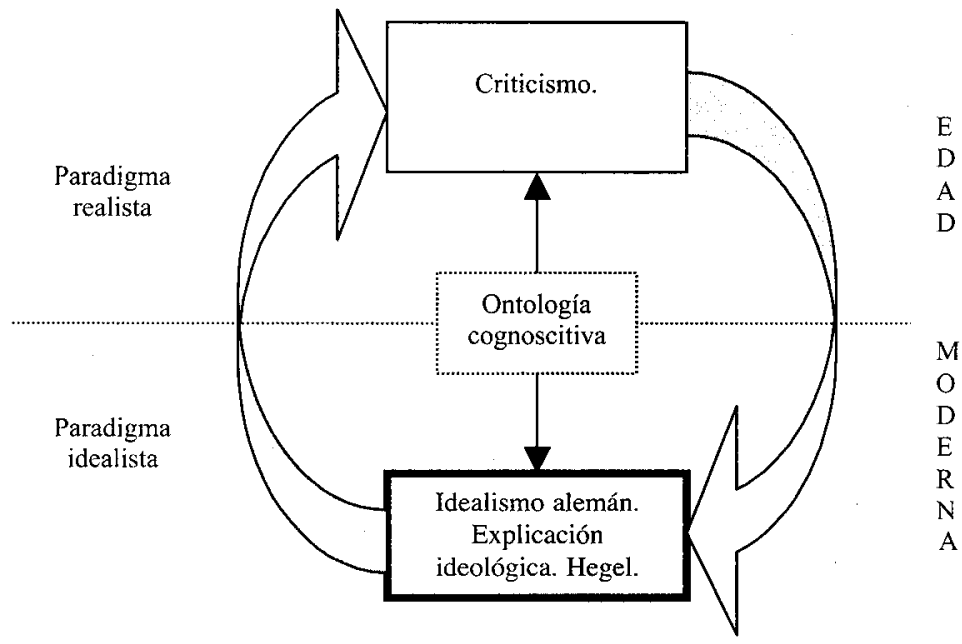

FINALES DEL SIGLO XVIII Y PRINCIPIOS DEL XIX

\section{La Epistemología idealista como investigación trascendental del conocimiento}

Efectivamente, frente a la filosofía positivista de la ciencia (Jiménez Ruiz, 2005: 286293), se fue fraguando principalmente en el ámbito alemán una tendencia antipositivista de carácter hermenéutico, basada en el rechazo del monismo metodológico, la física matemática como canon ideal regulador de toda explicación científica y el afán predictivo y causalista (Mardones, 1991: 30).

Esta Epistemología idealista será concebida posteriormente -ahora en la contemporaneidad- como una epistemología centrada en la investigación trascendental del conocimiento (Jiménez Ruiz, 1999: 289-290); será la realizada por el Idealismo romántico del historiador Droysen y el filósofo Schleiermacher a mediados del siglo XIX, el Historicismo de Dilthey a finales del xix, la Ontología fenomenológica de Heidegger y el Neocriticismo de Cassirer en la primera mitad del siglo xx, y, finalmente, el Relativismo epistemológico de Kuhn, Feyerabend y Lakatos - y actualmente Laudan— en la segunda mitad del siglo $\mathrm{xx}^{3}$.

3 Principalmente en la obra de Droysen, Grundriss der historik (1858); en las obras completas de Schleiermacher, Sämmtliche werke, edición de L. Jonas, Berlín, 1835-1864, en las que expone sus reflexiones sobre los problemas del conocimiento; en el ensayo de Dilthey sobre la formación de la hermenéutica (Die Entstehung der Hermeneutik), publicado en 1900 , en el que desarrolla su teoría de la comprensión histórica como fundamento de las ciencias del espíritu; en la obra de Heidegger El Ser y el Tiempo (Sein und Zeit), publicada en 1927, en la 


\section{INVESTIGACIÓN TRASCENDENTAL DEL CONOCIMIENTO}

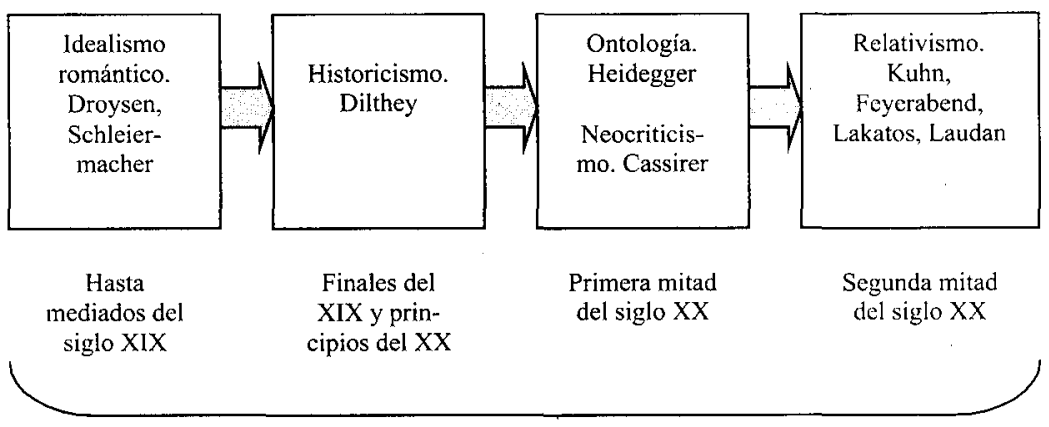

EDAD CONTEMPORÁNEA

\subsection{El programa de investigación idealista}

La aportación fundamental de estos hermeneutas idealistas consiste en precisar que las manifestaciones de lo singular son comprendidas como expresión de lo interior. En este sentido, no captar en una manifestación, conducta o hecho histórico esta dimensión interna equivale a no conocerlo (Mardones, 1991: 30).

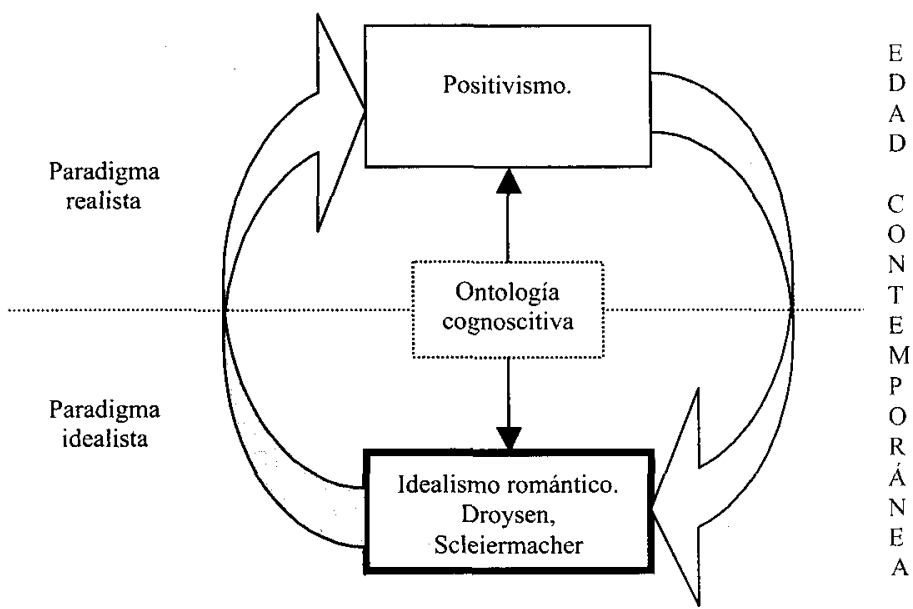

HASTA MEDIADOS DEL SIGLO XIX

que analiza la comprensión como elemento constitutivo existencial del ser en el mundo (Dasein); en El problema del conocimiento (1907), en el que Cassirer plantea su concepción del conocimiento como consecuencia de un contenido de conciencia preexistente; y finalmente en las obras de Kuhn, The Structure of Scientific Revolutions, publicada en 1962, con su teoría de los paradigmas; Feyerabend, Explanation, reduction and empiricism, publicada también en 1962 y «Against Method» en Minnesota Studies in the Philosophy of Science, iv, págs. 17-130, en 1970; y Lakatos, The Methodology of Scientific Reseach, de 1978. 
Droysen fue el primero en utilizar la distinción entre explicación y comprensión $n^{4}$, con el fin de precisar el método de la historia — la comprensión - frente al de la física matemática -la explicación-y al de la filosofía - el conocimiento-.

En este sentido, para Schleiermacher el conocimiento es la construcción de un entendimiento comparativo - propone lo que hay que entender como algo general, y encuentra lo particular al compararlo con otros, entendidos desde la misma perspectiva general-y adivinatorio - mientras se transforma en el otro, intenta buscar inmediatamente lo individual-, puesto que todo discurso tiene una doble operación con la totalidad del lenguaje y con el pensamiento total del su autor. De esta manera, el comprender se refiere tanto al lenguaje, como interpretación gramatical, cuanto a los hechos mentales, como interpretación psicológica.

Ello exige una metodología que, en el caso schleiermachiano, es simpatética, puesto que considera la interpretación como la forma de llegar al otro para saberlo.

\subsection{La importancia del historicismo}

Desde la perspectiva de los valores y la dialéctica entre las ciencias de la Cultura o el Espíritu ${ }^{6}$, Dilthey (1978) sostiene que el conocimiento se produce cuando se encuentran un yo y un tú que forman parte de un todo histórico cultural, gracias a la posibilidad de la comunicación.

Así, Dilthey acentuará además en las ciencias humanas la pertenencia del investigador y la realidad investigada al mismo universo histórico: el mundo cultural del hombre. Se trata, consecuentemente, de una unidad sujeto-objeto que permite el conocimiento desde dentro de los fenómenos históricos sociales (Mardones, 1991: 31).

En palabras de Dilthey (1980: 82):

«La Naturaleza nos es ajena. Pues es para nosotros algo externo, no interior. La sociedad es nuestro mundo. Presenciamos con toda la energía de nuestro ser entero, el juego de las interacciones dentro de ella, pues advertimos en nosotros mismos desde dentro, con la más viva inquietud, las situaciones y energías con que ella construye su sistema».

4 Así, a la explicación se le otorga competencia sobre fenómenos y procesos generales, frente a la comprensión, a la que le adjudica la esfera de lo singular; a la explicación se le supone atender a las causas, a la comprensión, la atención a las razones; en la explicación se halla implicada tan sólo la racionalidad del sujeto cognitivo, en la comprensión entran en juego otras dimensiones del sujeto, dimensiones de experiencia o vivencia y de familiarización; la explicación aparece como conocimiento desde fuera y en doble exterioridad, la comprensión como un conocimiento desde dentro y en interioridad; para explicar es preciso, primero experimentar u observar de manera controlada y sistemática y después analizar, inferir y deducir en una lógica correcta, incluida la lógica de la probabilidad, para llegar a la comprensión son otros los caminos, de ordinario más globales; el acercamiento explicativo tiende a tomar los hechos humanos como cosas, el comprensivo, incluso a la naturaleza no humana en términos (Gómez Caffarena y Mardones, 1992: 63-66).

5 Hasta tal punto Schleiermacher insiste en la importancia del autor que afirma que para comprender por ejemplo un texto debemos no sólo entrar en la mente del autor sino también identificarnos con él; cf. Bravo y Schökel, 1997: 27.

6 Puesto que, en el fondo, Dilthey establece y sistematiza la distinción entre los objetos naturales y los culturales; cf. Cuesta Abad, 1991: 109. 
Con su reflexión, Dilthey ha trasladado a la hermenéutica actual problemáticas como las de la historicidad de la comprensión, la consideración de la posición histórica en el proceso de comprensión, el papel de la distancia histórica, la historia de los efectos, etc. (Szondi, 1997: 64).

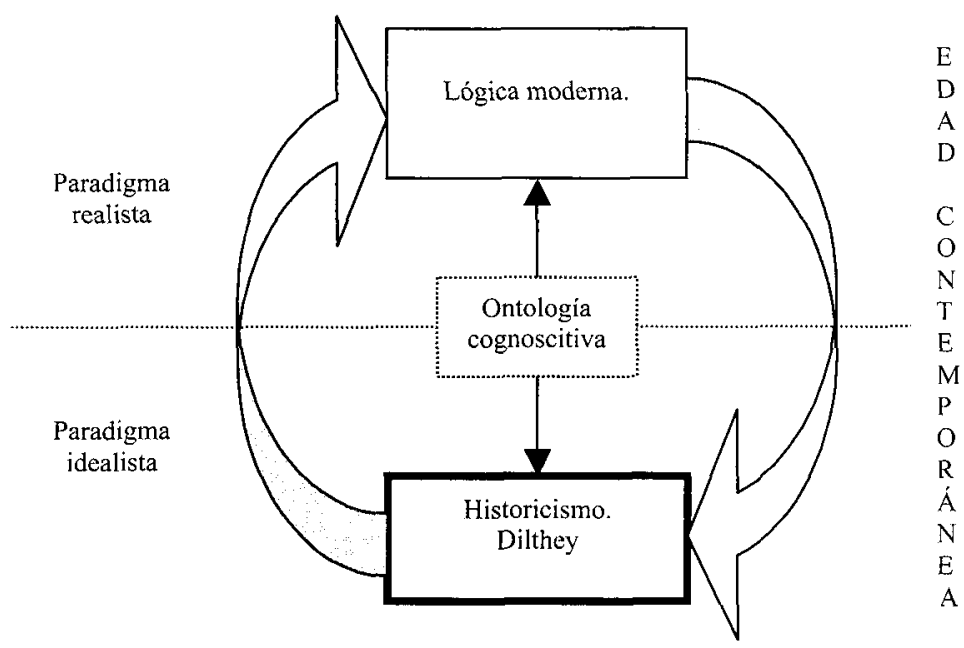

FINALES DEL XIX Y PRINCIPIOS DEL XX

En este caso, el quehacer del epistemólogo coincide con el quehacer del historiador puesto que éste ha de revivir la vivencia que sustenta la objetivación del espíritu en la historia (Ortiz Osés, 1973: 54-55). Se trata, pues, de una interpretación del conocimiento humano como una autocomprensión del hombre en su mundo ${ }^{7}$.

\subsection{La ontología fenomenológica}

Por otro lado, Heidegger supera el romanticismo schleiermachiano y el historicismo diltheyano elaborando una ontología fenomenológica de carácter universal. Sostiene que el lenguaje adquiere con la historia una determinada carga significativa que hay que descubrir. Por ello, la única solución consiste en trascender la realidad y hacer hablar al lenguaje en cuanto lenguaje para que, de esta manera, hombre y mundo puedan unirse (Lledó, 1970: 117 y ss.).

En este sentido, el entendimiento forma parte de la estructura misma del ser humano siendo, de esta manera, autocomprensión a partir del $\operatorname{ser}^{8}$. Esta comprensión se produce históricamente puesto que nuestro mundo histórico es una precondición de nuestra experiencia y, por tanto, forma parte de toda interpretación (Hirsch, 1997: 147).

7 Tanto la hermenéutica metódica de Schleiermacher como las tipologías de las concepciones del mundo de Dilthey han estado en la base de las distintas formulaciones sobre la comprensión e interpretación. Una reflexión al respecto puede verse en Sánchez Meca, 1997: 41-54.

8 Sobre la hermenéutica heideggeriana y sus consecuencias teóricas más relevantes puede verse Borges Duarte, 1995: 213-232. 
Así, la Epistemología tiene como objeto y sujeto a un tiempo el problema del conocimiento y la comprensión, entendida ésta como una realización exegética del mundo (significación y sentido) a través del lenguaje (Ortiz Osés, 1986: 226-228).

Por otro lado, para Cassirer (1971) el lenguaje tiene un papel activo que se manifiesta en la creación del mundo cultural. Por ello, tanto el lenguaje como el arte y el mito son funciones simbólicas.

Precisamente, el lenguaje es el que permite al hombre elevarse a la esfera de las intuiciones puras y, de allí, al plano superior de los conceptos y los juicios. Su axioma fundamental es, pues, que el símbolo crea su modelo, de manera que el conocimiento es una consecuencia de un contenido de conciencia preexistente (neokantismo).

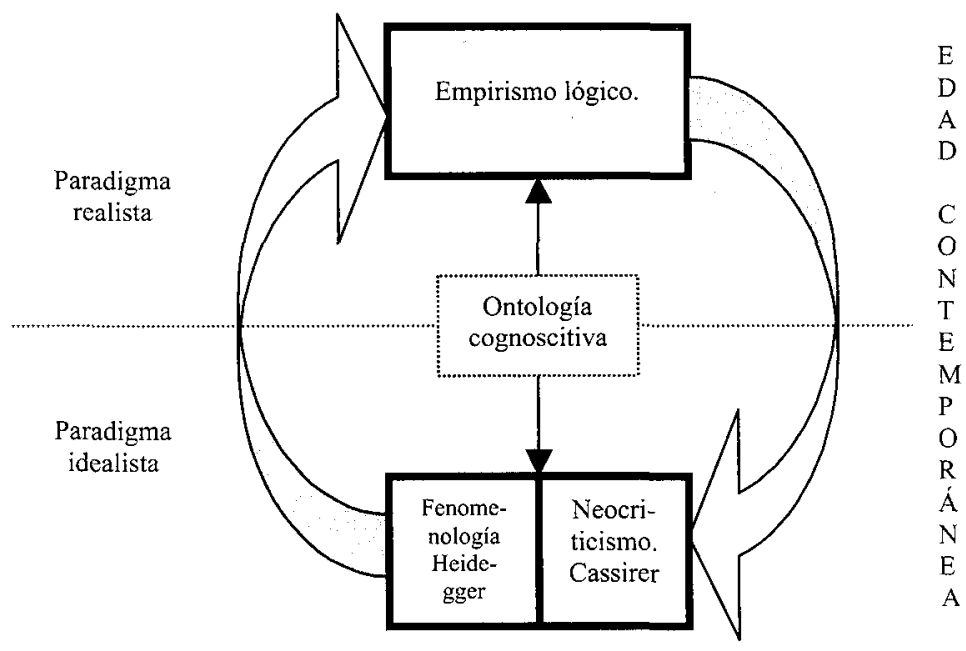

HASTA MEDIADOS DEL SIGLO XIX

\subsection{El Relativismo}

A partir de los años 60, la corriente historicista - siempre en contra de la Epistemología realista predominante, sobre todo la de Carnap y Popper - se constituye en una de las bases más sólidas de la Epistemología idealista.

Partiendo del énfasis en la relevancia de los estudios historiográficos (Díez y Moulines, 1999: 32), desprecian los métodos formales y proponen una imagen más amplia de la dinámica del conocimiento que la propuesta por los epistemólogos realistas.

En este sentido, podemos decir que la publicación en 1962 de La estructura de las revoluciones cientificas de Kuhn supuso la auténtica crisis del empirismo lógico - Paradigma realista - al sostener que la experiencia que los científicos tienen del mundo está en función de los paradigmas ${ }^{9}$ aceptados colectivamente por las comunidades científicas a las que pertenecen (Valor Yébenes, 2000: 18). 


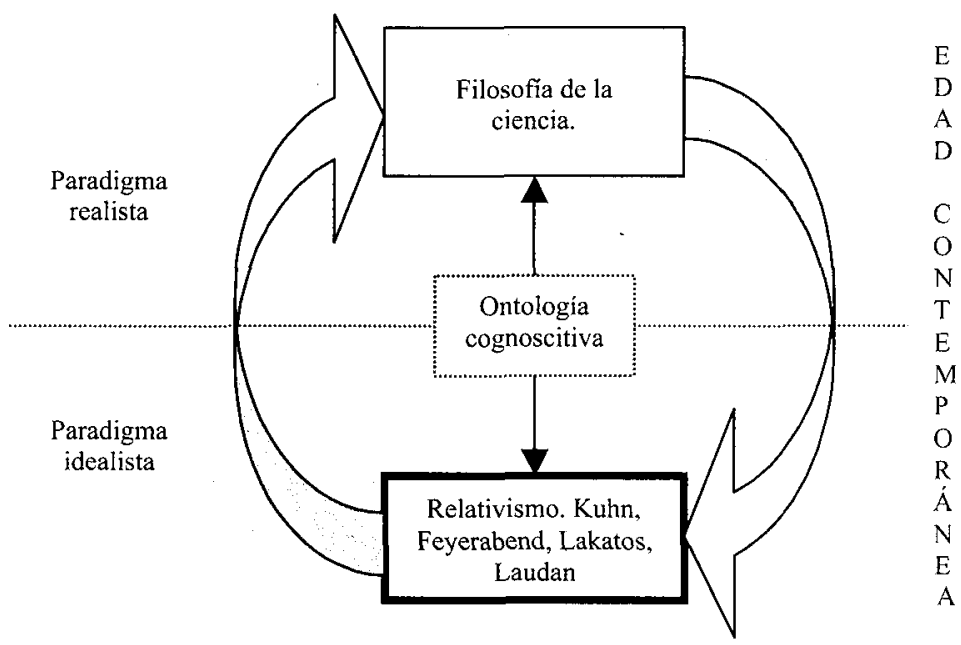

SEGUNDA MITAD DEL SIGLO XX

Dicho de otra forma, Kuhn sostiene que el conocimiento que tenemos del mundo exterior puede verse modificado radicalmente en función del paradigma que adoptemos, lo que nos lleva inevitablemente a un relativismo epistemológico que posibilitará posteriormente, en la década de los $70^{10}$, la concepción del conocimiento científico como un producto social; es la tesis principal de la sociología del conocimiento (Bunge, 2000: 225-262), desarrollada por la etnometodología, el relativismo y los estudios de ciencia y género, entre otros (Echeverría, 1995: 22-32).

Por tanto, como desarrollará Laudan posteriormente, lo importante ante cualquier norma metodológica es saber si tenemos o podemos encontrar evidencias mostrando que los medios propuestos en la norma promueven de manera mejor que sus rivales el objetivo cognitivo asociado a ellos (Gómez, 1995: 27).

Así pues, Kuhn para dar cuenta del cambio racional insiste, sobre todo, en la historia externa de la ciencia como motor del cambio científico, mientras que Laudan se apoya en la historia cognitiva que, a base de preguntas y respuestas, como cualquier otra actividad cognitiva, ha ido despejando incógnitas, resolviendo enigmas, eliminando anomalías, explicando hechos y clarificando conceptos, en suma, resolviendo problemas ${ }^{11}$.

10 Efectivamente, Kuhn ha tenido una gran repercusión en el ámbito de la Filosofía de la ciencia, en la segunda mitad del siglo xx. Sin embargo, ha sido la década de los 70 el período de años en el que más documentos fueron publicados sobre sus investigaciones, coincidiendo con una serie de obras publicadas por Kuhn y por conferencias que tuvieron por objeto la discusión que suscitó su teoría. Ello puede verse en el trabajo de Peral, Estévez y Pulgarín (1997: 623-635) en el que, a partir de una búsqueda retrospectiva de la producción literaria científica sobre la teoría kuhniana, escrita durante los años 1966-1995, en una serie de bases de datos internacionales, se llega a esta conclusión.

11 Un interesante trabajo en el que se comparan los puntos de vista de Kuhn y de Laudan sobre sus respectivos modelos para dar cuenta del cambio racional en la ciencia puede verse en Martínez de Velasco (1995: 199-210). En él, su autor nos muestra cómo Laudan, a partir de su modelo reticulado de racionalidad, en el que métodos, teorías y objetivos pueden cambiar de forma parcial y gradual, permite que podamos seguir hablando de cambio 
En este sentido, Lakatos (1974: 26 y ss.) se revela contra el que había sido su maestro (Popper), señalando que la teoría o la hipótesis central se nos muestra envuelta en una serie de teorías o visiones. Lakatos llamará programas de investigación cientifica a este conjunto formado por un centro firme, aceptado convencionalmente, y el cinturón de hipótesis auxiliares.

Como sostiene Mardones (1991: 51), los programas de investigación serán aceptables para Lakatos mientras sigan prediciendo (Lakatos, 1974: 28) nuevos hechos con éxito y explicándolos mejor que su rival.

Se trata, por tanto, de un «pensar a la contra», máxima que, según Feyerabend (1981) guía el quehacer científico. En efecto, la idea de un método con principios firmes, inalterables y absolutamente obligatorio en el quehacer científico presenta dificultades puesto que el progreso se ha producido mediante la infracción de determinadas reglas —a veces, incluso mediante pasos anarquistas ocasionales (Feyerabend, 1981: 11-12)-. Por ello, el científico debe adoptar una metodología pluralista comparando teorías con teorías, en lugar de hacerlo con experiencias y datos, esforzándose en mejorar en lugar de eliminar puntos de vista (Feyerabend, 1981: 31), poniendo al descubierto la racionalidad implícita en toda la historia de la ciencia (Laudan, 1986: 199 y ss.).

\section{La Epistemología idealista como investigación trascendente del lenguaje}

Estos estudios trascendentales sobre el conocimiento tienen también su parangón en lo que será ya propiamente la Epistemología centrada en la investigación trascendental del lenguaje, llevada a cabo, entre otros, por el Idealismo trascendental de Humboldt, en la $1^{\mathrm{a}} \mathrm{mitad}$ del siglo xIX, el Trascendentalismo existencialista de Gadamer después de la $2^{\mathrm{a}}$ Guerra Mundial, y el mentalismo lingüístico y la Lingüística transformac̉ional, también en el siglo xx.

\section{INVESTIGACIÓN TRASCENDENTAL DEL LENGUAJE}

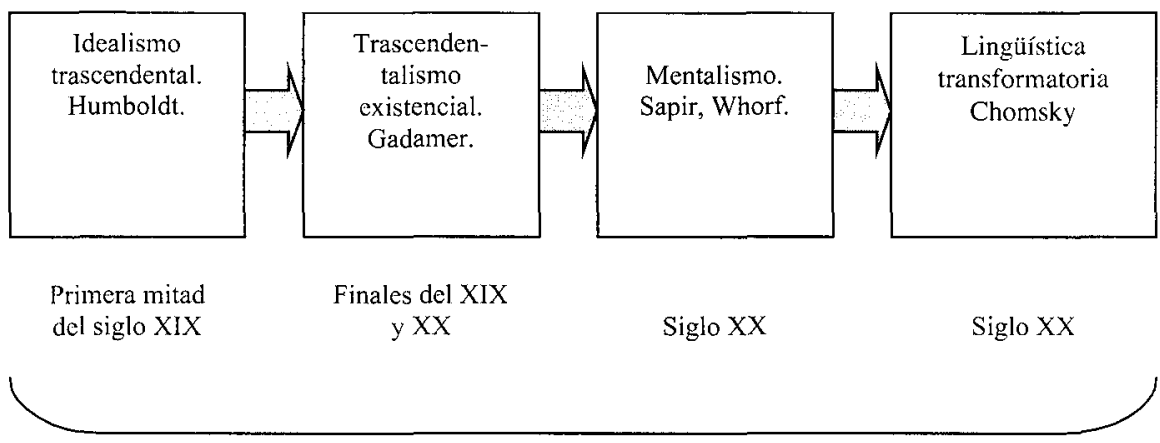

EDAD CONTEMPORÁNEA

científico progresivo, simplemente cuando una teoria o conjunto de teorías de una tradición de investigación son más eficaces que otras en la resolución de problemas. Laudan, por el contrario, cree que la ciencia, globalmente considerada, sigue siendo una empresa racional que cambia progresivamente, aunque puede ocurrir que haya elementos no racionales en la elección de una teoría. 


\subsection{La respuesta lingüística al cientificismo}

Humboldt es considerado en la Historia de la Lingüística (Robins, 1992: 195-200) como un gran teórico del lenguaje cuya gran aportación consistió en oponer a la Lingüística diacrónica dominante una Lingüística sincrónica cuya culminación fue la clasificación lingüística tipológica.

Sin embargo, debemos coincidir con Galán Rodríguez (1994: 167) en que:

«Este modo de presentación es a todas luces parcial y selectivo, pues Humboldt es percibido como filósofo y, por tanto, se tiende a separar su filosofía del lenguaje de su lingüística sin comprender que Humboldt no es un filósofo lingüista sino un lingüista filósofo [la cursiva es nuestra]».

Por ello, situamos a Humboldt en lo que hemos denominado Epistemologia idealista centrada en la investigación trascendental ya específicamente del lenguaje.

Para Humboldt, el lenguaje no es ni una visión del mundo (Weltanschauning) ni una concepción del mundo (Weltbild), sino un mundo intermedio mediador (Zwischenwelt). E1 lenguaje se concibe, así, como el auténtico entendimiento (a la vez subjetivo y objetivo) de la realidad, realizado gracias a la fuerza del espíritu creador humano que se objetiva y autoencuentra de un modo individual y social a la vez, en el lenguaje (Jiménez Ruiz, 1994: 196).

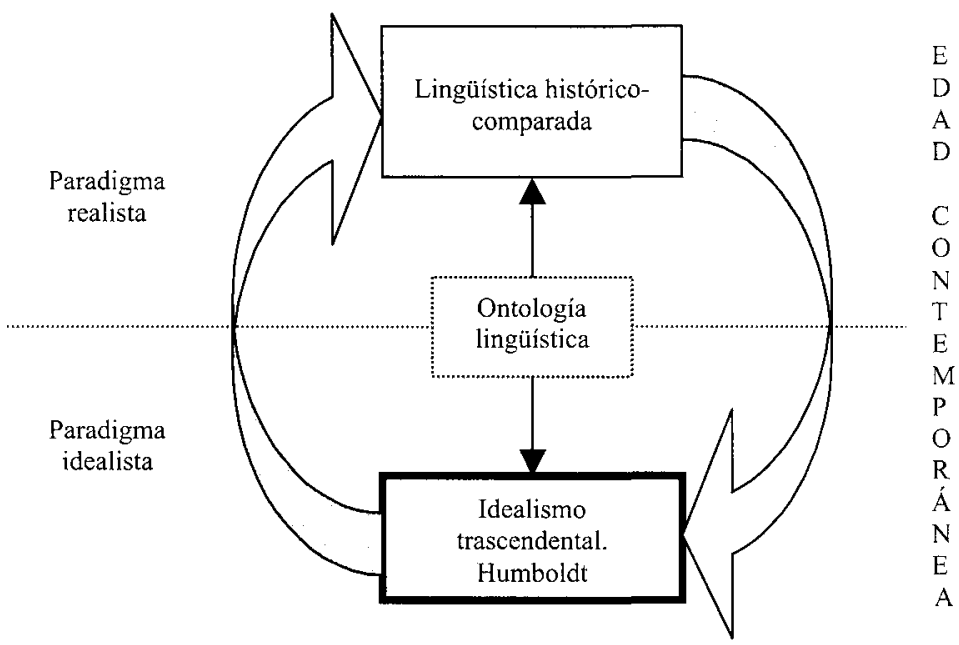

PRIMERA MITAD DEL SIGLO XX 
Estas dimensiones diferentes han llevado al lenguaje a instaurarse como sujeto de distintas disciplinas, según sea la faceta de él que predomine; a saber, de la lingüistica, si lo consideramos como ergon, de la psicología del lenguaje, si lo hacemos como dynamis y, finalmente, de la filosofía, si lo consideramos ahora como energeia. Con ello, son dos los principios que pueden establecerse del planteamiento humboldtiano:

- lenguaje como actividad, como creación del que habla, siendo al mismo tiempo subjetivo (puesto que no es algo dado por el mundo exterior, sino un modo peculiar de representar en nosotros ese mundo), y objetivo (ya que es obra de una nación a lo largo de su historia y, por consiguiente, extraño al individuo);

- y, finalmente, lenguaje como organismo, ya que, de hecho, no se trata sólo de un medio para expresar la Verdad, sino de un camino para descubrir aquello que no conocemos (Humboldt, 1990: 65 y ss.).

Así pues, frente a la concepción positivista del lenguaje como objeto mediador de la realidad inmediata, el lenguaje aparece en Humboldt como sujeto constitutivo de la realidad mediata. Con ello se inicia decisivamente la tradición idealista trascendental del lenguaje, continuada hoy por Weisgerber.

\subsection{El trascendentalismo lingüístico de Gadamer}

Podemos considerar a H. G. Gadamer como el padre de la hermenéutica trascendental del lenguaje actual ${ }^{12}$; fue defensor de la escuela de Heidegger - de hecho, fue discípulo de éste-, profesor de la Universidad de Heidelberg, director de la revista Philosophische Runschau y presidente de Congreso alemán de Filosofía.

Su reflexión consiguió trasladar la Epistemología desde la Gnoseología a la Ontología del lenguaje, inclinando la balanza del lado de la energeia humboldtiana (o lenguaje como energía del espíritu subjetivo humano) frente al ergon (o lenguaje como obra material o formal del hombre). Por ello, todo análisis debe serlo del lenguaje, ya que éste es el único medio para que se produzca el conocimiento y, para Gadamer, el conocimiento $-\mathrm{y}$, por tanto, el entendimiento- es siempre interpretativo - incluido el explicativo o científico-naturalista-. Es lo que Gadamer (Koselleck y Gadamer, 1997: 104) denomina «lingüisticidad»:

«La lingüisticidad que la hermenéutica emplaza en el centro no es sólo la de los textos; por tal entiende igualmente la condición del ser fundamental de todo actuar y crear humanos [la cursiva es nuestra]». 


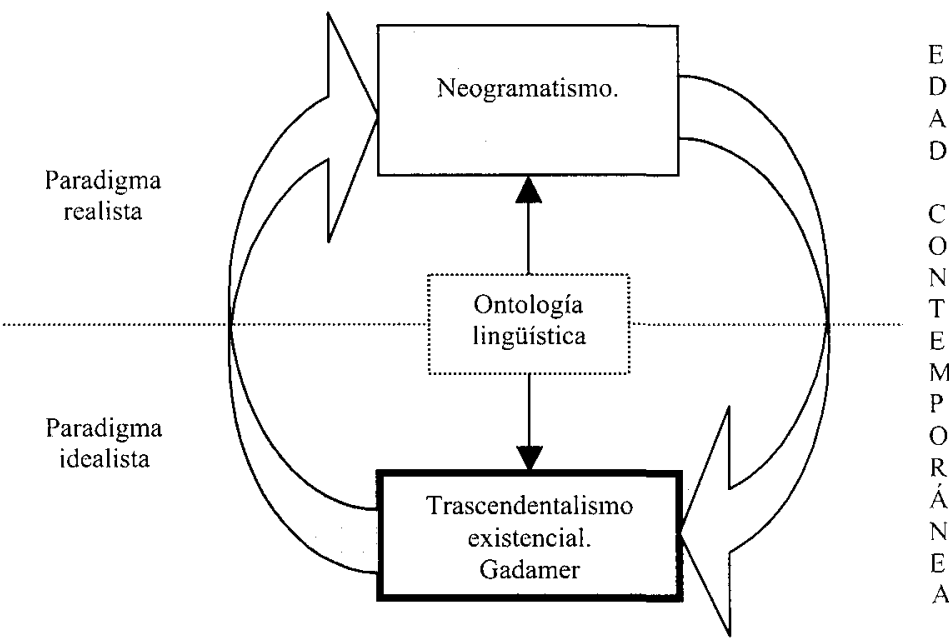

FINES DEL XIX Y SIGLO XX

El ser humano, por tanto, no trata de comprender el mundo, puesto que comprender es entenderse en el mundo. Trata, pues, de entenderse con los otros, de entender al otro a partir de las lenguas. Así, el lenguaje es el medio en el que se realiza el acuerdo de los interlocutores y el consenso sobre la cosa (Gadamer, 1977: 461).

Por ello, debe emplearse la verdadera productividad del lenguaje para entendernos, ya que, además de su concreción como un sistema de reglas, constituye la base social del pensamiento individual, formando parte tanto de la cultura - puesto que es un comportamiento humano aprendido sobre la base de la capacitación biológica del hombre (Gadamer, 2000: 17) - como de la estructura social - ya que permite la comunicación en el espacio y en el tiempo-.

«En esto consiste primordialmente hablar y la hermenéutica sirve para desarrollar la posibilidad de transmitir al otro lo que uno piensa de verdad y obtener de él la respuesta, la réplica de su modo de pensar» (Koselleck y Gadamer, 1997: 122).

Se trata, por tanto, de alcanzar el entendimiento gracias al diálogo, lo que quiere decir que se entiende el mundo no como ser - definido en última instancia como idea- sino como lenguaje -definido en última instancia como comunicación- (Ortiz Osés, 1973: 116).

La consecuencia será para Gadamer (1977: 467-468) que la hermenéutica deberá elaborar un lenguaje común como medio universal en el que se realice la comprensión misma, entendida ésta no como un desplazamiento al interior del otro reproduciendo sus vivencias sino - como hemos dicho antes - como un consenso sobre la cosa. 


\subsection{El mentalismo lingüístico}

Para el mentalismo, las palabras no son objetos reales sino mentales, por ello, no son símbolos de percepciones aisladas, sino símbolos de conceptos. El significado, así presentado, sería una idea asociada a un significante en la mente de los hablantes, un reflejo perceptivo desprovisto de toda intencionalidad, un esquema o pauta de relación del individuo con su mundo, entendiéndose la percepción no como un conjunto de percepciones aisladas sino como una sensación del cuerpo con el ambiente. Todo ello se traduce en una concepción del lenguaje como vehículo de transmisión del pensamiento.

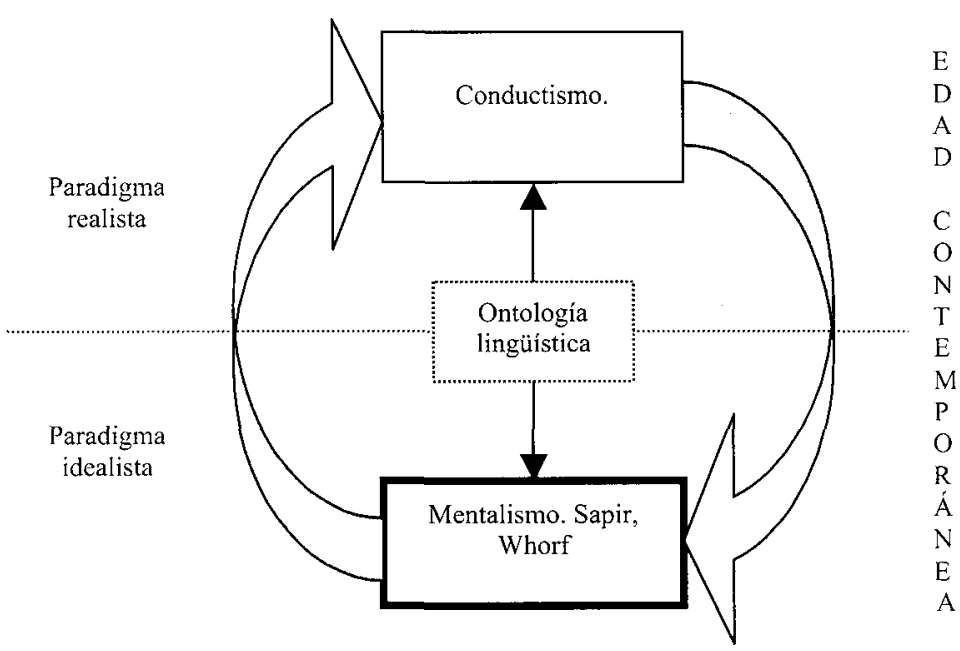

SIGLO XX

Se produce, pues, una identificación entre lenguaje y pensamiento, que es más bien un presupuesto materialista (que Marx y Engels dejan claro en la Ideología alemana). Por otra parte, la posición de los filósofos del lenguaje alemanes (Humboldt, Herder, Harmann) aunque implica una cierta coincidencia entre lenguaje y Weltanschauung, está teñida de un claro relativismo lingüístico.

La identidad origina la hipótesis de Sapir-Whorf o teoría de la relatividad lingüística: la cultura (aquello que la sociedad hace o piensa) no coincide con la lengua que dicha cultura utiliza como medio de expresión. Nos encontramos en un nuevo principio de la relatividad que sostiene que todos los observadores no son conducidos por la misma evidencia fisica o la misma representación del universo, a menos que sus bases lingüísticas sean similares o puedan ser calibradas de alguna manera (Whorf, 1973: 212 y ss.). Así, la teoría de la relatividad lingüística defiende que el lenguaje es un factor creativo que modifica la visión del mundo de los hablantes de acuerdo con la categoría de relación con que se caracteriza el pensamiento. 
A pesar de las críticas recibidas por Whorf basadas principalmente en el hecho de que toda su teoría se centró en la estructura gramatical y sobre las categorías que esta estructura impone en el pensamiento y en el comportamiento (Ullmann, 1968: 256-257), sin tener en cuenta que el vocabulario posee también una organización y una estructura propia; y en el hecho de que las estructuras gramaticales básicas de una lengua permanecen constantes a lo largo de su desarrollo durante largos periodos de tiempo en los que la cultura cambia radicalmente (Kutschera, 1979: 354); esta hipótesis es la primera reformulación en nuestro siglo de la identidad racionalista lenguaje y pensamiento (Gutiérrez López, 1975: 147-150; Miller, 1989: 135-138; Schaff, 1967: 85-138).

\subsection{El Generativismo como prototipo del Paradigma idealista de la Lingüística}

Finalmente, vamos a concluir este breve panorama histórico señalando que este idealismo cognoscitivo ha tenido su desarrollo lingüístico más importante — puesto que su formulación se considera como prototipo del Paradigma idealista de la Lingüística - en el Generativismo chomskyano, desarrollado en EE. UU., a partir del Racionalismo cartesiano y del Idealismo trascendental humboldtiano, principalmente.

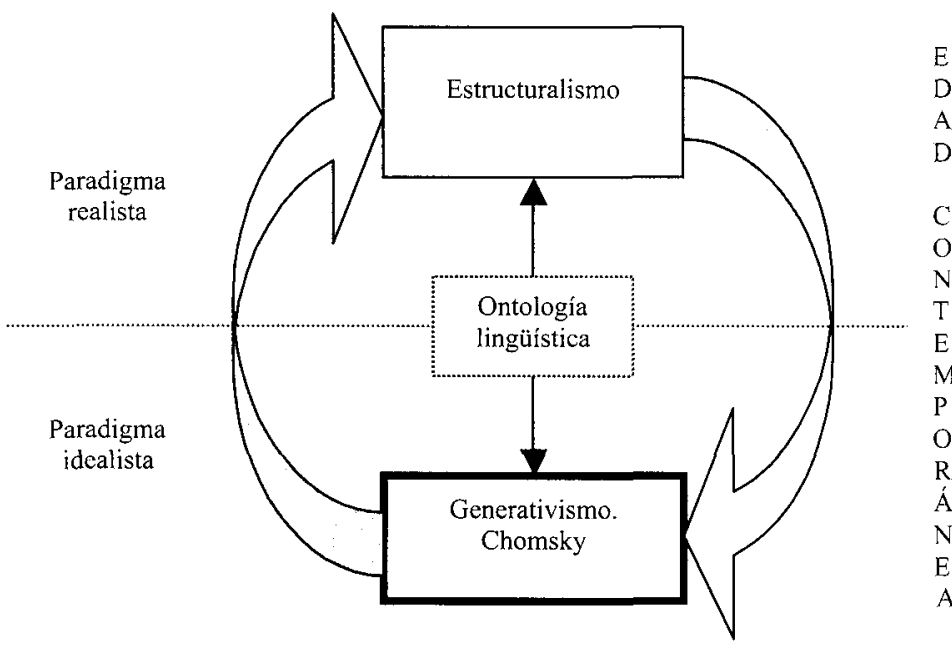

SIGLO XX

La propuesta chomskyana aparece frente al ambiente conductista en el ámbito psicológico, empirista lógico en el ámbito filosófico, y estructuralista distribucional en el ámbito estrictamente lingüístico.

Quizá su gran revolución estuvo en oponerse a los tres.

Por ello, vamos a concluir este breve panorama representando ahora en un gráfico la evolución del Paradigma Idealista. 


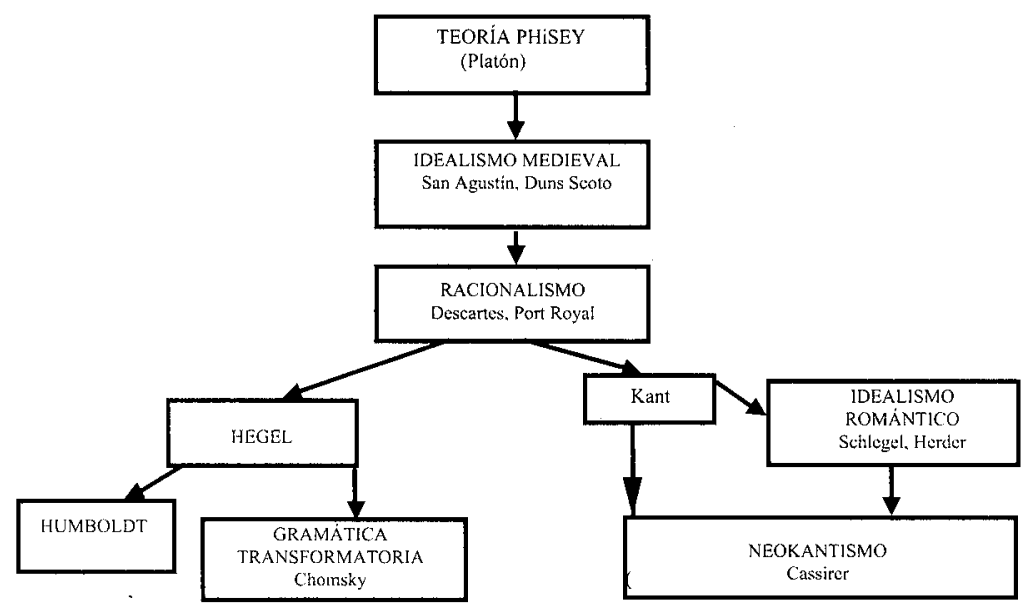

\section{Referencias bibliográficas}

Bravo, J. M. y L. A. Schökel (1997): Apuntes de hermenéutica. Madrid, Trotta.

Borges Duarte, I. (1995): “¿Recepción o interpretación? Reflejos de la mirada heideggeriana hacia

Kant", Anales del Seminario de Historia de la Filosofia, 12, págs. 213-232.

Bunge, M. (2000): La relación entre la Sociologia y la Filosofia. Madrid, Edaf.

Cassirer, E. (1971): Filosofia de las formas simbólicas. México, F. C. E.

Cuesta Abad, J. M. (1991): Teoria hermenéutica y literaria. Madrid, Visor.

Descartes, R. (1990): Discurso del método. Madrid, Tecnos.

Díez, J. A. y C. U. Moulines (1999): Fundamentos de Filosofia de la Ciencia. Barcelona, Ariel.

Dilthey, W. (1978): El Mundo Histórico. México, F. C. E.

Dilthey, W. (1980): Introducción a las ciencias del espiritu. Madrid, Alianza.

Echeverría, J. (1995): Filosofía de la Ciencia. Madrid, Akal.

Esteban Ortega, J. (1996): "La revitalización hermenéutico-lingüística de la memoria en H. G. Gadamer y E. Lledó”, Pensamiento, 52, págs. 403-428.

Feyerabend, P. K. (1981): Tratado contra el método. Madrid, Tecnos.

Gadamer, H. G. (1977): Verdad y método. Salamanca, Sígueme.

Gadamer, H. G. (2000): Elogio de la Teoría. Barcelona, Península.

Galán Rodríguez, C. (1994): "La teoría lingüística de Wilhelm von Humboldt", Anuario de Estudios Filológicos, xVII, págs. 165-185.

Gómez, R. J. (1995): "Racionalidad: epistemología y ontología". En Olivé, L. (ed.): Racionalidad epistémica. Trotta, Madrid, págs. 19-40.

Gómez Caffarena, J. y J. M. Mardones (coords.) (1992): Cuestiones epistemológicas. Barcelona, Anthropos.

Gutiérrez López, G. (1975): Estructura del lenguaje y conocimiento. Hacia una epistemología de la semiótica. Madrid, Fragua.

Hegel, G. W. (1964): La Fenomenologia del Espirtu. México, F. C. E.

Hirsch, E. (1997): "Tres dimensiones de la hermenéutica". En Domínguez Caparrós, J. (ed.): Hermenéutica. Madrid, Arco/Libros, págs. 137-158.

Humboldt, W. (1990): Sobre la diversidad de la estructura del lenguaje humano. Barcelona, Anthropos. 
Jiménez Ruiz, J. L. (1994): Semántica sintomática. Propuestas para una hermenéutica comprensiva del sentido. Alicante, Universidad de Alicante.

Jiménez Ruiz, J. L. (1999): "Lenguaje e historia: A propósito de la controversia entre Kosellek y Gadamer", Estudios de Lingiḯstica, 13, págs. 281-295.

Jiménez Ruiz, J. L. (2001): Iniciación a la Lingüística. Alicante, Editorial Club Universitario.

Jiménez Ruiz, J. L. (2004): “ La historiografía como vertiente diacrónica de la epistemología de la lingüística: propuestas de caracterización". En Corrales Zumbado et alii (eds.): Nuevas aportaciones a la Historiografia lingüística. Actas del IV Congreso Internacional de la SEHL. Madrid, Arco/Libros, págs. 895-907.

Jiménez Ruiz, J. L. (2005): "La problemática de la continuidad a través de la ruptura: los cambios interteóricos en los programas de investigación realista de la Lingüística”, ELUA, 19, págs. 275 299.

Koselleck, R. y H. G. Gadamer (1997): Historia y hermenéutica. Barcelona, Paidós, I. C. E., Universidad Autónoma de Barcelona.

Kuhn, T. S. (1981): La estructura de las revoluciones cientificas. México, F. C. E.

Kutschera, F. von (1979): Filosofia del lenguaje. Madrid, Gredos.

Lakatos, I. (1974): Historia de la ciencia y sus reconstrucciones racionales. Tecnos, Madrid.

Laudan, L. (1986): El progreso y sus problemas: hacia una teoría del crecimiento cientifico. Madrid, Encuentro.

Lledó, E. (1970): Filosofia y Lenguaje. Barcelona, Ariel.

López Sáenz, Ma C. (1996): "El sentido de la Historia de la Filosofía para la Filosofía Hermenéutica", Contrastes, 1, págs. 151-172.

Mardones, J. M. (1991): Filosofia de las ciencias humanas y sociales. Materiales para una fundamentación científica. Barcelona, Anthropos.

Martínez de Velasco, J. (1995): "El cambio científico y el modelo de solución de problemas de L. Laudan", Themata, 13, págs. 199-210.

Miller, G. A. (1989): Lenguaje y habla. Madrid, Alianza.

Monserrat, J. (1984): Epistemología evolutiva y teoria de la ciencia. Madrid, Universidad Pontificia de Comillas.

Ortiz Osés, A. (1973): Antropología hermenéutica. Madrid, Editorial Ricardo Aguilera.

Ortiz Osés, A. (1986): La nueva filosofia hermenéutica. Barcelona, Anthropos.

Peral, D., Estévez, P y A. Pulgarín (1997): "Presencia del pensamiento kuhniano en la literatura científica: 1966-1995", Llull. Boletín de la Sociedad española de Historia de las ciencias, 39, págs. 623-635.

Ramos Requejo, R. (1995): "Para una teoría de la interpretación textual: de Gadamer a Habermas", Grial, 33, págs. 61-93.

Revilla Guzmán, C. (1995): "Los límites de la textualidad. Escritura y diálogo en la hermenéutica gadameriana", Anales del Seminario de Metafisica, 29, págs. 171-188.

Robins, R. H. (1992): Breve historia de la Lingüística. Madrid, Paraninfo.

Sánchez Meca, D. (1997): "Comprensión e interpretación de las obras filosóficas", Diálogo filosófico, 37, págs. 41-54.

Schaff, A. (1967): Lenguaje y conocimiento. México, Grijalbo.

Szondi, P. (1997): "Introducción a la hermenéutica literaria". En Domínguez Caparrós, J. (ed.): Hermenéutica. Madrid, Arco/Libros, págs. 59-74.

Ullmann, S. (1968): Lenguaje y estilo. Buenos Aires, Paidós.

Valor Yébenes, J. A. (2000): Metodología de la investigación cientifica. Madrid, Biblioteca Nueva.

Vilana Taix, V. (1995): "La relación lenguaje-mundo en Verdad y método", Studium, 1, págs. 261271 .

Whorf, B. L. (1973): Lenguaje, pensamiento y realidad. Barcelona, Seix Barral. 